\title{
$\checkmark$ Research Square

\section{Microbiological Profile, Antimicrobial Susceptibility, and Outcome Among Patients Admitted to Addis Ababa Intensive Care Units; a Cross-sectional Study.}

Yemane Gebremedhin ( $\sim$ yeman31@gmail.com )

St. Paul's Hospital Millennium Medical College

Menbeu Sultan

St. Paul's Hospital Millennium Medical College

Dagmawi Tesfaye

St. Paul's Hospital Millennium Medical College

\section{Research Article}

Keywords: microbiological profile, antimicrobial susceptibility, outcome, Intensive Care Unit

Posted Date: December 30th, 2021

DOI: https://doi.org/10.21203/rs.3.rs-1178576/v1

License: (c) (i) This work is licensed under a Creative Commons Attribution 4.0 International License.

Read Full License 


\section{Abstract}

Background: ICU infections are major health care problems affecting millions globally each year. ICU mortality of infectious patients is increasing and as high as $14.31 \%$ to $45.4 \%$. The objective of this study was to determine microbiological culture profile, their antimicrobial susceptibility and treatment outcome of patients admitted to intensive care units.

Methods and materials: An institutional based cross sectional study was conducted in all patients with microbiological culture and susceptibility results after admission to intensive care units from January 2019 to December 2019. A modified, pretested questionnaire was used to collect data and analyzed using SPSS version 25. Correlation and regression analysis was done for association. $\mathrm{P}$ value of $<0.05$ with $\mathrm{Cl}$ of $95 \%$ was taken as clinically significant.

Results: A total of 106 patients with 173 culture results were analyzed. Majority 68(64.2\%) were males and the mean age of the patients were $35.08 \pm 1.6$ years. The most common source of infection was the pulmonary system $84(54.5 \%)$ followed by urinary tract infection $26(16.9 \%)$. Forty-four (25.43\%) of cultures had growth. Gram negative microorganisms were identified in $35(68.63 \%)$ of isolates and Acinetobacter species accounts for $10(28.57 \%)$ followed by Klebsiella pneumonia and E. coli $7(13.725 \%)$ each. Generally, higher resistance was observed with cephalosporins and penicillins, whereas sensitivity to carbapenems, PIP/TAZ, and amikacin was satisfactory. The mortality associated with intensive care infections was found to be $32.1 \%$.

Conclusion: This study revealed that the mortality rate associated with ICU infection was $32.1 \%$. Early identification, targeted provision of antimicrobials based on microbiological culture and susceptibility result has to be implemented to have better outcome. Designs of future multicenter and large studies are crucial to improve outcome of critically ill patients.

\section{Introduction}

Intensive care unit infections are among the most serious infections and leading causes of morbidity and mortality in hospitalized patients, where indiscriminate and inadequately prolonged use of antimicrobials is common and leads to emerging proliferation of resistant strains $(1,2)$.

Notable among these resistant microorganisms are methicillin-resistant s. aureus (MRSA), and vancomycin-resistant enterococcus (VRE) which pose increased mortality and morbidity $(4,5)$.

Knowledge of antimicrobial susceptibility in ICU is crucial and far more important for giving effective treatment and decreasing the spread of drug resistant microorganisms. And it is crucial step for early intervention, decrease health-care costs, improve outcome of patients and increase treatment provider satisfaction. 
Nevertheless, there was no study done in the ICU setting, both institutionally and country wise. So, this study on the assessment of microbiological culture profile, antimicrobial susceptibility and outcome of ICU patients will be a great input in addressing the gap.

\section{Objectives}

The objective of this study was to determine microbiological culture profile, their antimicrobial susceptibility and treatment outcome of patients admitted to intensive care units of Saint Paul's hospital millennium medical college and AaBET hospital ICUs.

\section{Methods And Materials}

Institutional based cross-sectional study was conducted at Saint Paul's hospital millennium medical college and AaBET hospital ICUs. SPHMMC is a referral teaching hospital in Addis Ababa, Ethiopia. And AaBET hospital is a branch of SPHMMC in Addis Ababa, Ethiopia which is an emergency complex which gives services for emergency, burn and trauma cases. SPHMMC has 14 bedded ICU and AaBET hospital has 11 bedded ICU. Both are general ICUs admitting both surgical and medical patients.

Data was collected from patients chart using pretested and structured questionnaire, after receiving ethical clearance from the college's institutional review board. All microbiological culture and susceptibility results of patients sent after intensive care unit admission from January 2019 to December 2019 were studied. Data was collected by trained data collectors using pretested standardized checklists developed by the principal investigator. Socio-demographic data, comorbidities, sites of infection, causative micro-organisms, ICU admission category, associated organ failure, type of culture sent, antimicrobials given prior to culture sampling and based on susceptibility result, and patient outcome to ICU was retrieved. All data was taken from patient record chart with collected laboratory culture and susceptibility results after selecting their MRN from ICU registration book.

Data was entered and statistical analysis performed using SPSS version 25. Chi-square test was used for categorical variables and mean, median and standard deviation (SD) for continuous variables. Associated risk factors were determined using logistic regression and correlation.

\section{Results}

\subsection{Demographic characteristics}

A total of 106 patients having culture result after admission to ICU over a period of one year were analyzed. Majority 68(64.2\%) were males and the median age was 30.5 years, within the range of 3-82 years. Most of the patients $75(70.8 \%)$ were admitted from the emergency department. The median length of ICU stay was 31 days (interquartile range of 3-128 days). And the hospital length of stay prior to ICU admission was $6.89 \pm 10.52$ (mean \pm SD). More than $99 \%$ of the patients have met the SIRS criteria. 


\subsection{Clinical characteristics of patients}

Underlying comorbidities were identified in $49(46.2 \%)$ of patients. The most common comorbidity was hypertension $14(28.6 \%)$ followed by coronary artery disease and congestive heart failure $10(20.4 \%)$ combined. Majority of deaths $22(64.70 \%)$ were associated with comorbidity. Coronary artery disease and congestive heart failure being the most common comorbidity $6(17.6 \%)$ followed by hypertension $5(14.70 \%)$. The most common site of infection was the pulmonary system $84(54.5 \%)$ followed by urinary tract infection 26(16.9\%), skin/soft tissue infection 18(11.7\%), CNS 16(10.4\%), and 10(6.5\%) intraabdominal focus. Pulmonary and urinary focus was higher in male patients 55(65.47\%) and 18(69.23\%) respectively, whereas intra-abdominal focus was higher in females $9(90 \%)$. Organ failure occurred in majority $94(88.7 \%)$ of patients, with the respiratory system comprised $84(44.7 \%)$ of organ failures followed by neurologic system 60(31.9\%).

As shown in Table 1. 
Outcome to ICU (discharge and death) among patients admitted to SPHMMC and AaBET ICUs from January 2019 to December 2019 with regard to comorbidity, infection site, and organ failure ( $N=$ number of patients).

\begin{tabular}{|c|c|c|c|c|c|}
\hline Variable & Total $(\mathrm{N}=106)$ & Discharge $(\mathrm{N}=65)$ & $P$ value & Death $(\mathrm{N}=34)$ & $P$ value \\
\hline Comorbidity & $49(46.2 \%)$ & & & $22(64.7 \%)$ & \\
\hline RVI & $3(6.1 \%)$ & $1(1.5 \%)$ & .317 & $2(5.9 \%)$ & .196 \\
\hline DM & $8(16.3 \%)$ & $5(7.7 \%)$ & .944 & $3(8.8 \%)$ & .735 \\
\hline HTN & $14(28.6 \%)$ & $8(12.3 \%)$ & .733 & $5(14.7 \%)$ & .757 \\
\hline $\mathrm{CAD} / \mathrm{CHF}$ & $10(20.4 \%)$ & $4(6.15 \%)$ & .149 & $6(17.6 \%)$ & .047 \\
\hline COPD/Asthma & $2(4.1 \%)$ & $1(1.5 \%)$ & .743 & $1(2.9 \%)$ & .588 \\
\hline CKD & $6(12.2 \%)$ & $3(4.6 \%)$ & .562 & $3(8.8 \%)$ & .338 \\
\hline CLD & $1(2.0 \%)$ & - & .210 & $1(2.9 \%)$ & .146 \\
\hline Malignancy & $2(4.1 \%)$ & - & .073 & $1(2.9 \%)$ & .588 \\
\hline Infection site & 154 & 95 & & 59 & \\
\hline Pulmonary & $84(54.5 \%)$ & $51(53.7 \%)$ & .804 & $27(45.8 \%)$ & .977 \\
\hline Urinary & $26(16.9 \%)$ & $18(18.9 \%)$ & .345 & $4(6.8 \%)$ & .036 \\
\hline CNS & $16(10.4 \%)$ & $9(9.5 \%)$ & .655 & $7(11.9 \%)$ & .282 \\
\hline Skin/soft tissue & $18(11.7 \%)$ & $9(9.5 \%)$ & .283 & $9(15.25 \%)$ & .075 \\
\hline Gastrointestinal & $10(6.5 \%)$ & $8(8.4 \%)$ & .206 & $2(3.4 \%)$ & .395 \\
\hline Organ failure & 188 & 105 & & 71 & \\
\hline Neurologic & $60(31.9 \%)$ & $33(31.4 \%)$ & .129 & $23(32.4 \%)$ & .117 \\
\hline Respiratory & $84(44.7 \%)$ & $48(45.7 \%)$ & .086 & $30(42.25 \%)$ & .119 \\
\hline Renal & $31(16.5 \%)$ & $17(16.2 \%)$ & .383 & $13(18.3 \%)$ & .165 \\
\hline Cardiovascular & $13(6.9 \%)$ & $7(6.7 \%)$ & .559 & $5(7.0 \%)$ & .603 \\
\hline
\end{tabular}

RVI- Retroviral Infection, DM- Diabetes Mellitus, HTN- Hypertension, CAD/CHF- Coronary artery disease/congestive heart failure, COPD- Chronic Obstructive Pulmonary Disease, CKD-Chronic Kidney Disease, CLD-Chronic Liver Disease, CNS- central nervous system.

\subsection{Microbiological profile and antimicrobial susceptibility}

Out of 173 cultures analyzed $84(48.55 \%)$ were blood cultures, urine 69(39.88\%), pus 6(3.47\%), CSF and body fluid $5(2.89 \%)$ each, and $2(1.16 \%)$ of the cultures were tracheal aspirate and stool culture each. Forty four $(25.43 \%)$ of cultures had growth of 51 microorganism isolates. Gram negative microorganisms 
were identified in 35(68.63\%) of isolates (Table 2) and Acinetobacter species accounts for $10(28.57 \%)$ followed by Klebsiella pneumonia and E. coli 7(13.725\%) each and proteus mirabilis 4(7.84\%). Ten (19.60\%) of microorganisms were gram positive microorganisms, of which $5(50 \%)$ were coagulase negative staphylococci, 2(20\%) enterococci and staphylococcus aureus each and 1(10\%) Streptococcus pneumoniae. Six (11.76\%) of the microorganisms grown were fungal, all from urine culture (Figure 1).

Acinetobacter species had shown sensitivity to meropenem 5(55.6\%), ceftazidime 1(50\%), amikacin $3(42.9 \%)$, gentamicin 2(40\%), amoxicillin/clavulanate and cotrimoxazole 1, 2(33.3\%) respectively, and ciprofloxacin and tobramycin $2,1(25 \%)$ respectively, but resistant at all test times to other cephalosporins (cefazoline, ceftriaxone, cefepime, cefotaxime), TTC, nitrofurantoin and piperacillin.

Klebsiella pneumoniae was sensitive to imipenem 1(100\%), piperacillin/tazobactam 1(50\%), chloramphenicol $1(50 \%)$, meropenem $3(42.9 \%)$, ciprofloxacin $1(20 \%)$, but resistant at all test times to all cephalosporins (cefuroxime, ceftazidime, cefepime, and cefotaxime), nitrofurantoin, ampicillin, and gentamicin.

Escherichia coli had shown sensitivity to meropenem 4(100\%), amikacin 1(100\%), chloramphenicol and nitrofurantoin 2(100\%) each, gentamicin 4(80\%), piperacillin/tazobactam 1(50\%), ciprofloxacin 2(33.3\%), and TMP/SMX 1(25\%). Whereas, resistant to cephalosporins (cefazoline, cefotaxime, and cefuroxime), augmentine, piperacillin and tetracycline at all test times.

Pseudomonas aeruginosa was sensitive to ciprofloxacin 2(100\%), amoxicillin, cefepime and piperacillin $1(100 \%)$ each, meropenem and tobramycin $1(50 \%)$ each.

Table 2

Type of microorganism isolates on microbiological culture among patients admitted to SPHMMC and AaBET ICUs from January 2019 to December 2019 (N=number of microorganism isolates).

\begin{tabular}{|c|c|c|c|c|c|}
\hline \multirow[t]{3}{*}{ Type of culture } & \multicolumn{5}{|c|}{ Number of Microorganism isolates $(\mathrm{N}=51)$} \\
\hline & $\begin{array}{l}\text { Gram negative } \\
(\mathrm{N}=35)\end{array}$ & $\begin{array}{l}\text { Percentage } \\
\%(68.7)\end{array}$ & $\begin{array}{l}\text { Gram positive } \\
(\mathrm{N}=10)\end{array}$ & $\begin{array}{l}\text { Percentage } \\
\%(19.6)\end{array}$ & $\begin{array}{l}\text { Fungus } \\
(\mathrm{N}=6)\end{array}$ \\
\hline & & & & & $11.7 \%$ \\
\hline Blood & 5 & 9.80 & 7 & 13.73 & - \\
\hline Urine & 23 & 45.10 & 1 & 1.96 & 6 \\
\hline Pus & 3 & 5.90 & 2 & 3.92 & - \\
\hline Body fluid & 1 & 1.96 & - & - & - \\
\hline Tracheal aspirate & 3 & 5.90 & - & - & - \\
\hline
\end{tabular}


Microorganisms isolated from urine culture which were resistant to all tested antimicrobials were like Klebsiella ozaena (ceftriaxone, gentamicin, nitrofurantoin, piperacillin, cotrimoxazole, and tobramycin), and rhizobium radiobacter (ampicillin, amoxicillin/clavulanate, ceftriaxone, cefepime, ciprofloxacin, nitrofurantoin, TTC, cotrimoxazole, and tobramycin).

A single citrobacter species isolated from urine culture was only sensitive to amikacin, but resistant to cephalosporins (ceftriaxone and cefuroxime), tmp/smx, ciprofloxacin, gentamicin, amox/clav, TTC, and piperacillin. And also enterococcus species from urine culture had shown sensitivity to ciprofloxacin, daptomycin, and vancomycin, intermediate activity against erythromycin, but resistant to penicillin $\mathrm{G}$.

Proteus mirabilis from urine cultures were sensitive to levofloxacin 1(100\%), piperacillin/tazobactam 2(66.7), intrapenem 1(50\%), gentamicin 1(33.3\%), and intermediate to tobramycin 1(50\%). But, resistant to ciprofloxacin 4(100\%), ceftriaxone 4(100\%), tmp/smx 4(100\%), and meropenem 1(100\%).

Generally, higher resistance was observed with cephalosporins and penicillins, whereas sensitivity to carbapenems, PIP/TAZ, and amikacin was satisfactory (Table 3) and (Figure 2). 
Table 3

Antimicrobial resistance and sensitivity frequency among patients admitted to SPHMMC and AaBET ICUs from January 2019 to December 2019 ( $\mathrm{N}=$ number of tests).

Antimicrobials $\quad$ Resistance: N (\%) Sensitive: N (\%)




\begin{tabular}{|c|c|c|}
\hline Antimicrobials & Resistance: N (\%) & Sensitive: N (\%) \\
\hline Ampicillin & $10(100 \%)$ & - \\
\hline Amikacin & $6(42.8 \%)$ & $8(57.2 \%)$ \\
\hline Amoxacillin/Clavulanate & $12(80 \%)$ & $3(20 \%)$ \\
\hline Ceftriaxone & $14(100 \%)$ & - \\
\hline Cefotaxime & $10(100 \%)$ & - \\
\hline Cefepime & $11(91.7 \%)$ & $1(8.3 \%)$ \\
\hline Cefuroxime & $4(100 \%)$ & - \\
\hline Ceftazidime & $5(71.4 \%)$ & $1(14.3 \%)$ \\
\hline Cefazoline & $4(100 \%)$ & - \\
\hline Ciprofloxacin & $23(76.7 \%)$ & $7(23.3)$ \\
\hline CAF (chloramphenicol) & $5(62.5 \%)$ & $3(37.5 \%)$ \\
\hline Clindamycin & $1(50 \%)$ & $1(50 \%)$ \\
\hline Daptomycin & - & $2(100 \%)$ \\
\hline Erythromycin & $1(33.3 \%)$ & - \\
\hline Gentamycin & $10(52.6 \%)$ & $8(42.1 \%)$ \\
\hline Imipenem & $1(16.7 \%)$ & $4(66.7 \%)$ \\
\hline Intrapenem & $1(33.3 \%)$ & $2(66.7 \%)$ \\
\hline Meropenem & $4(25 \%)$ & $11(68.7 \%)$ \\
\hline Nitrofurantoin & $13(86.7 \%)$ & $2(13.3 \%)$ \\
\hline Norfloxacin & $1(50 \%)$ & $1(50 \%)$ \\
\hline Oxacillin & $2(100 \%)$ & - \\
\hline Penicillin & $2(100 \%)$ & - \\
\hline Piperacillin & $9(81.8 \%)$ & $2(18.2 \%)$ \\
\hline Piperacillin/Tazobactam & $2(18.2 \%)$ & $6(54.6 \%)$ \\
\hline SMX/TMP (cotrimoxazole) & $19(86.4 \%)$ & $3(13.6 \%)$ \\
\hline TTC (Tetracycline) & $9(90 \%)$ & $1(10 \%)$ \\
\hline Tobramycin & $10(62.5 \%)$ & $3(18.7 \%)$ \\
\hline Vancomycin & $2(66.7 \%)$ & - \\
\hline
\end{tabular}


A single staphylococcus aures isolated from pus culture shown sensitivity to gentamicin, vancomycin, clindamycin, and TMP/SMX, but resistant to penicillins, piperacillin, and cloxacillin.

Enterococcus species had shown sensitivity against vancomycin 2(100\%), daptomycin 1(100\%), pip/taz $1(100 \%)$, imipenem $1(100 \%)$, and ciprofloxacin $1(50 \%)$. Intermediate activity against erythromycin; but resistant to amikacin, cephalosporins (ceftriaxone and cefotaxime), amox/clav, piperacillin, and ampicillin/sulbactam.

Streptococcus pneumoniae from blood culture was only sensitive to pip/taz, and resistant to penicillins (amoxicillin, amikacin, and amox/clav), cephalosporins (ceftriaxone, ceftazidime, and cefepime), and nitrofurantoin.

A single klebssiella species from blood culture wassensitive to amox/clav, ciprofloxacin, gentamicin, TTC, imipenem, and norfloxacin. But, resistant against cephalosporins, piperacillin, and TMP/SMX.

Staphylococcus aureus from pus culture was sensitive to cotrimoxazole, vancomycin and clindamycin but resistant to oxacillin, penicillin, and ciprofloxacin.

\subsection{Antimicrobial use and outcome of patients to ICU}

Almost all patients, $103(97.3 \%)$ took antimicrobials before ICU admission or prior to culture sampling, and ceftriaxone was used in 67(62.3\%) patients, followed by vancomycin in $62(58.5 \%)$, and metronidazole in 56(52.8\%) patients. The most common indications were pulmonary infection in $68(37.2 \%)$, followed by empiric therapy in $26(14.2 \%)$, and urosepsis in $25(13.7 \%)$ patients (Table 4 ).

Fourteen (13.2\%) patients took meropenem based on culture and susceptibility result, followed by ciprofloxacin $3(2.8 \%)$, and cotrimoxazole and gentamycin in 2(1.9\%) patients each.

Sixty five (61.3\%) patients were discharged, whereas thirty four (32.1\%) patients died during their ICU stay. And patients with urinary tract infection and septic shock before admission were significantly associated with higher risk of death. 
Table 4

Comparison among outcome to ICU and indication for antimicrobial use before admission or prior to culture sampling among patients admitted to SPHMMC and AaBET ICUs from January 2019 to December 2019.

\begin{tabular}{|llllll|}
\hline Indication & $\mathbf{N}(\%)$ & Death & P value & Discharge & P value \\
\hline - Bacterial sepsis & $14(13.2 \%)$ & $3(21.4 \%)$ & .364 & $9(64.3 \%)$ & .809 \\
- Septic shock & $5(4.7 \%)$ & $1(20 \%)$ & .019 & $4(80 \%)$ & .053 \\
- CNS infection & $11(10.4 \%)$ & $6(54.5 \%)$ & .093 & $5(45.5 \%)$ & .258 \\
- Urosepsis & $25(23.6 \%)$ & $5(20 \%)$ & .036 & $16(64 \%)$ & .086 \\
- Pulmonary & $68(64.2 \%)$ & $24(35.3 \%)$ & .347 & $39(57.4 \%)$ & .266 \\
- Intra-abdominal & $8(7.5 \%)$ & $1(12.5 \%)$ & .221 & $7(87.5 \%)$ & .255 \\
- Soft-tissue/skin & $16(15.1 \%)$ & $7(43.75 \%)$ & .282 & $9(56.3 \%)$ & .655 \\
- Infective endocarditis & $1(0.9 \%)$ & - & .495 & - & .430 \\
- Empiric & $26(24.5 \%)$ & $6(23.1 \%)$ & .262 & $18(69.2 \%)$ & .345 \\
- Prophylaxis & $9(8.5 \%)$ & $2(22.2 \%)$ & .513 & $7(26.9 \%)$ & .294 \\
\hline
\end{tabular}

\section{Discussion}

Majority of the patients were males 68(64.2\%) similar to a study by Dahmesh et. al. (51.4\%), but different from a study done in septic shock patients at TASH at which $60 \%$ were females $(24,34)$.

The mean age and median length of stay in the ICU was $35.8 \pm 1.6$ years and 31 days respectively, different from TASH septic shock patients' $47.99 \pm 16.5$ years and 4 days respectively. And, most of our patients $(70.8 \%)$ were admitted directly from the emergency, $>99 \%$ met SIRS criteria different from a study done in TASH which revealed in only $60 \%$ of patients (34). It could be because AaBET hospital is a trauma center, and trauma victimizes the young, also a reason for prolonged ICU admission.

Underlying comorbidity was found in $49(46.2 \%)$ of patients, hypertension being the most common comorbidity in 14(28.6\%), lower than and different from TASH ICU septic shock patients which revealed $89.5 \%$ associated comorbidity and immunosuppression being the most common in $33.9 \%$ of patients and that of US study which showed $55.5 \%$ comorbidity with COPD accounting $12.3 \%(34,40)$. This can be from epidemiologic shift that NCDs are affecting the young.

Organ failure occurred in majority (88.7\%) of our patients, which were lower than TASH study in septic shock patients, but the most frequently flailing organs were similar (34). This can be from the nature of our patients, which are not only septic shock patients, and the prolonged ICU stay.

Of the 106 patients studied 173 cultures sent were analyzed, and only $44(25.43 \%)$ of cultures had growth of microorganisms with 51 isolates, which was similar with a study in eastern Ethiopia hospitals with 
isolation rate of $27.9 \%$, but higher than that of a study done in TASH $16.5 \%$ and lower than a study done in Nepal 39.6\% $(32,33,34)$.

The most common source of infection was the pulmonary and urinary tract similar with a study in five ICUs of Imam Reza hospital and TASH ICU septic shock patients, but different from Jimma university hospital Ali et.al. from which surgical site infection being the most common source $(29,34,36)$.

Pulmonary and urinary focus was higher in male patients 55(65.47\%) and $18(69.23 \%)$ respectively, while intra-abdominal focus was higher in females $9(90 \%)$ in our study. Also a study in organisms causing UTI in ICU patients of Kenyatta national hospital reveal higher incidence in male patients (31).

Gram negatives were the most commonly identified microorganisms similar with other studies, but the isolated species were different $(32,33,34)$.

And patients with $\mathrm{CAD} / \mathrm{CHF}$, urinary tract source of infection and septic shock before admission had greater risk of death which was different from other studies which revealed high risk of death with different factors such as infection with acinetobacter species, renal replacement therapy, use of mechanical ventilation, COPD, malignancy, pulmonary cause of infection and antibiotic use before admission (39-44).

The study found $32.1 \%$ mortality with ICU infections which was similar to other different studies 14.31 $45.4 \%$, lower than that of an African study $47.2 \%(17-22,39)$.

High antibiotic resistance rate $(>60 \%)$ was observed with ciprofloxacin, cephalosporins, piperacillin, cotrimoxazole, chloramphenicol, tetracycline, nitrofurantoin, tobramycin and penicillins including oxacillin similar to a study in Chad-Ndjamena general hospital and other studies $(26,27,28,30)$. Good sensitivity $(>65 \%)$ was shown with carbapenems and vancomycin, dabtomycin $(100 \%)$ with two tests, amikacin $(57.2 \%)$, and piperacillin/tazobactam (54.5\%) similar with other studies $(26,28,30,31,32)$.

Almost all patients 103(97.3\%) studied took antimicrobials before ICU admission, which contributes to antimicrobial resistance that was directly proportional to the volume of antimicrobials consumed (13).

Whereas, the use of antimicrobial based on culture and susceptibility result showed only at $13.2 \%$ which needs improvement to have better outcome, reduce adverse events and their attendant costs, and prevent the emergence and dissemination of resistant microorganisms (12).

\section{Strengths And Limitations}

Data was collected retrospectively, which predisposed to lack of complete and clear data especially with the duration of antimicrobials provided before and after ICU admission, no clear date of culture sampling, and onset of infection. It was studied in different two institutional, multidisciplinary ICU settings which makes it a strong study. 


\section{Conclusion And Recommendation}

Our study had shown patients with urinary source of infection, septic shock, and patients with comorbid illness especially coronary artery disease or congestive heart failure had significant risk of death. Resistant gram negatives were the predominant microorganisms identified which pose greatest challenges to ICU quality of care and prolonged ICU stay complications.

Designs of future multicenter and large studies are crucial to improve outcome of critically ill patients and targeted provision of antimicrobials based on microbiological culture and susceptibility results has to be implemented.

\section{Abbreviations}

AaBET- Addis-Ababa Burn Emergency and Trauma Hospital, CSF- Cerebro Spinal Fluid, ICU- Intensive and Critical care Unit, MRN- Medical Registration Number, SIRS- Systemi Inflammatory Response Syndrome, SPHMMC-Saint Paul`s Hospital Millennium Medical College, TASH- Tikur Anbessa Specialized Hospital.

\section{References}

1. Kollef MH, Fraser VJ. Antibiotic resistance in intensive care unit setting. Ann Intern Med. 2001; 134:298-314.

2. Shankar PR, Partha P, Dubey AK, Mishra P, Deshpande VY. Intensive care unit drug utilization in a teaching hospital in Nepal. Kathmandu Univ Med J. 2005; 3: 130-137.

3. Tripathi KD. Essentials of Medical Pharmacology.6th ed. New Delhi: Jaypee Brothers. 2009; 667-681.

4. Safdar N, Maki DG. The commonality of risk factorsfor nosocomial colonization and infection withantimicrobial resistant Staphylococcus aureus, Enterococcus, Gram negative bacilli,Clostridiumdifficile and Candida. Ann InternMed. 2002; 136: 834-844.

5. Kumarasamy KK, Toleman MA, Walsh TR, Bagaria J, Butt F, Balakrishnan R, Chaudhary, Doumith M, Giske CG, IrfanS.Emergence of a new antibiotic resistance mechanism in India, Pakistan, and the UK: a molecular, biological, and epidemiological study. Lancet Inf Dis. 2010; 10: 597-602.

6. Lovely B, Kaniz F, J Ashrafu H, Mohammad OF, ASM Areef, Md. Abu HG Morshed, Md. Belayet H. Bacterial profile and their antimicrobial resistance pattern in an intensive care unit of a tertiary care Hospital in Dhaka. Ibrahim Med. Coll. J. 2010; 4(2): 66-69.

7. Bhadade RR. Prospective evaluation and mortality outcome of nosocomial infections in medical intensive care unit at tertiary care teaching center in Mumbai. Int J Res Rev. Oct 2013; Vol 05(19).

8. Kennedy AM, Elward AM, Fraser VJ. Survey of knowledge, beliefs, and practices of neonatal intensivecare unit healthcare workers regarding nosocomial infections, central venous catheter care, and handhygiene. Infect. Control Hosp. Epidemiol. 2004; 25:747-52.

9. WHO. Report on the Burden of Endemic Health Care-Associated Infection Worldwide. Geneva, Switzerland: WHO; 2011. Available 
from:http://www.who.int/about/licensing/copyright_form/en/index.html.

10. Hall A. Editorial: nosocomial infection in developing countries: time to learn. Trop Med Int Health. 1998; 3(7):517-517.

11. Lul R. prevention and control of hospital-related infections in low and middle income countries. The open infectious disease journal. 2010; vol. 4, 125-131.

12. Shlaes DM, Gerding DN, John JF (Jr), Craig WA, Bornstein DL, Duncan RA, Eckman MR, Farrer WE, Greene WH, Lorian V. Society for Healthcare Epidemiology of America and Infectious Diseases Society of America Joint Committee on the Prevention of Antimicrobial Resistance: Guidelines for The prevention of antimicrobial resistance in hospitals. ClinInfect Dis. 1997; 25: 584-599.

13. Sharma PR, Barman P. Antimicrobial consumption and impact of "Reserve antibiotic indent form" in an intensive care unit. Indian J Pharmacol. 2010; 42: 301-305.

14. Radji M, Fauziah S, Aribinuko N. Antibiotic sensitivity pattern of bacterial pathogens in the intensive care unit of Fatmawati Hospital, Indonesia. Asian Pacific Journal of Tropical Biomedicine. 2011; 1:39-42.

15. Tullu MS, Deshmukh CT, Baveja SM. Bacterial profile, antimicrobial susceptibility pattern in catheter related nosocomial infections. J Postgrad Med. 1998; 44:7-13.

16. Vincent JL, Rello J, Marsall J, Silva E, Anzeuto A, Martin CD. International study of prevalence and outcomes of infection in intensive care units. JAMA. 2009; 302:2323-9.

17. Ak O, Batirel A, Ozer S, Çolakoğlu S. Nosocomial infections and risk factors in the intensive care unit of a teaching and research hospital: a prospective cohort study. Med SciMonit.2011; 17: PH29-34.

18. Ustun C, Hosoglu S, Geyik MF, Parlak Z, Ayaz C The accuracy and validity of a weekly pointprevalence survey for evaluating the trend of hospital acquired infections in a university hospital in Turkey. Int J Infect Dis. 2011; 15: e684-687.

19. Madani N, Rosenthal VD, Dendane T, Abidi K, Zeggwagh AA. Health-care associated infections rates, length of stay, and bacterial resistance in an intensive care unit of Morocco: findings of the International Nosocomial Infection Control Consortium (INICC). Int Arch Med.2009; 2: 29.

20. Sax H, Pitter D; Swiss-NOSO Network. Intercostal differences in nosocomial infection rates: importance of case-mix adjustment. Arch InternMed.2002; 162: 2437-2442.

21. Habibi S, Wig N, Agarwal S, Sharma SK, Lodha R. Epidemiology of nosocomial infections in medicine intensive care unit at a tertiary care hospital in northern India. Trop Doct. 2008; 38: 233-235.

22. Rizvi MF, Hasan Y, Memon AR, Abdullah M, Rizvi MF. Pattern of nosocomial infection in two intensive care units of a tertiary care hospital in Karachi. J Coll Physicians Surg Pak.2007; 17: 136-139.

23. Shalini S, Kranthi K, Gopal Krishna BK. The microbiological profile of nosocomial infections in the intensive care unit. J ClinDiagn Res. 2010; 4:3109-12.

24. Dahmash NS, Arora SC, Fayed DF, Chowdhury MN. Infections in critically ill patients: experience in MICU at a major teaching hospital. Infection. 1994; 22: 264-270. 
25. Chaitali P, Sunil KP, Pratyay PD, Parbaty P. A study on antibiotic sensitivity pattern of bacterial isolates in the intensive care unit of a tertiary care hospital in Eastern India. IntJ Basic ClinPharmacol. 2013 Apr; 2(2):153-159.

26. Kaushal VS, Tejas K Patel, Saklainhaider S Malek, Tripathi. Antibiotic Sensitivity Pattern of Bacterial Isolates from the Intensive Care Unit of a Tertiary Care Hospital in India. Trop J Pharm Res. December 2012; 11(6):991.

27. Trupti B, G. Shrivastava, G. S. Bhatambare, A. B. Deshmukh, V. Chitnis. Microbiological profile of lower respiratory tract infections in neurological intensive care unit of a tertiary care center from Central India. Journal of Basic and Clinical Pharmacy. June-August 2013; Vol. 4, Issue 3.

28. Deepti C, Avinash L, K. Sadawarte, Tukaram P. Microbiological Profile and Antimicrobial Sensitivity Pattern of Endotracheal Tube Aspirates of Patients in ICU of a Tertiary Care Hospital in Bhopal, India. Int.J.Curr.Microbiol.App.Sci.2017; 6(3):891-895.

29. H. Hamishehkar, P. Shadmehr, A. Mahmoodpoor, S. O. Mashayekhi, T. Entezari-Maleki. Antimicrobial susceptibility patterns among bacteria isolated from intensive care units of the largest teaching hospital at the northwest of Iran. Brazilian Journal of Pharmaceutical Sciences.2016; vol. 52, n. 3.

30. Michel K, Amon TD, Julius MN. Bacteriological profile and antimicrobial susceptibility patterns of urine culture isolates from patients inNdjamena, Chad. Pan African Medical Journal. 2017; 28:258.

31. Isabell M. Bacterial profile and antimicrobial susceptibility pattern of isolates causing urinary tract infections in critical care unit patients at Kenyatta national Hospital in Kenya (Nairobi). Available from: https://www.semanticscholar.org/paper

32. Sapkota J, Mishra B, Jha B, Sharma M. Bacteriological profile and their antimicrobial susceptibility pattern in central venous catheter tip culture. Journal of Pathology of Nepal.2017; Vol. 7, 1059-1061.

33. IbssaA, Solomon G. Common Bacterial Pathogens and Their Antimicrobial Susceptibility PatternsIn Patients with Symptomatic Urinary Tract Infections at Hiwot Fana and Jugal Hospitals in Eastern Ethiopia (Harar). Galore International Journal of Health Sciences and Research. April-June 2018; Vol.3, Issue 2.Website: www.gijhsr.com

34. Teklemichael H. Assessment of incidence and treatment outcome of septic shock among patients admitted to adult intensive care unit of Tikur Anbessa specialized hospital in Ethiopia (Addis Ababa): AAU; 2017-06.

35. Walelegn WY, Abera K, Feleke MY. Point prevalence of hospital-acquired infections in two teaching hospitals of Amhara region in Ethiopia. Drug, Healthcare and Patient Safety. 23 August 2016.

36. Solomon A, Melkamu B, Sisay B, Gebre K, Lule T, Yonas Y, Yesuf A, Netsanet F, Henok A, Mulatu G, Esayas KG. Health care associated infection and its risk factors among patients admitted to a tertiary hospital in Jimma University, Ethiopia. Antimicrobial Resistance and InfectionControl. 2018; 7:2. DOI 10.1186/s13756-017-0298-5.

37. Tsegaye A, Mulubrahan A, Enkosilassie Mengistu H. The burden of antimicrobial resistance at tertiary care hospital, southern Ethiopia: three years' retrospective study. BMC Infectious Diseases. 2019; 19:585. 
38. Zwer F. Definition, Incidence and Evolution of Sepsis and Septic Shock. Journal of Anesthesia and Critical Care. 2016; volume 5, issue 1.

39. Yasser S, Ulrich J, Xavier W, Tamas S, Jeffery L, Silvio AN, Ignacio ML, Mark L, Mary NL, JeanLV. Sepsis in Intensive Care Unit patients: world wide data from ICON audit. Oxford university press on behalf of Infectious Disease Society of America. 2018.

40. Angus DC, Linde-Zwirble WT, Lidicker J, Clermont G, Carcillo J, Pinsky MR. Epidemiology of severe sepsis in the United States: analysis of incidence, outcome, and associated costs of care. Critical Care Medicine-Baltimore. 2001; 29(7):1303-10.

41. Zhou J, Qian C, Zhao M, Yu X, Kang Y, Ma X. Epidemiology and outcome of severe sepsis and septic shock in intensive care units in mainland China. PloS one. 2014; 9(9):e107181.

42. Van GA, Bakker J, Veraart CP, van Hout BA. Prevalence and incidence of severe sepsis in Dutch intensive care units. Critical Care. 2004; 8(4):R153.

43. Annane D, Aegerter P, Jars-Guincestre MC, Guidet B. Current epidemiology of septic shock: the CUBRea Network. American journal of respiratory and critical care medicine. 2003; 168(2):165-72.

44. Meliha M, Ayse W, Sigdem C, Kamil T. Intensive Care Unit-Acquired infections: incidence, risk factors, and associated mortality in a Turkish University Hospital. Jpn. J. infect. Dis. 2005; 58, 297-302.

\section{Figures}

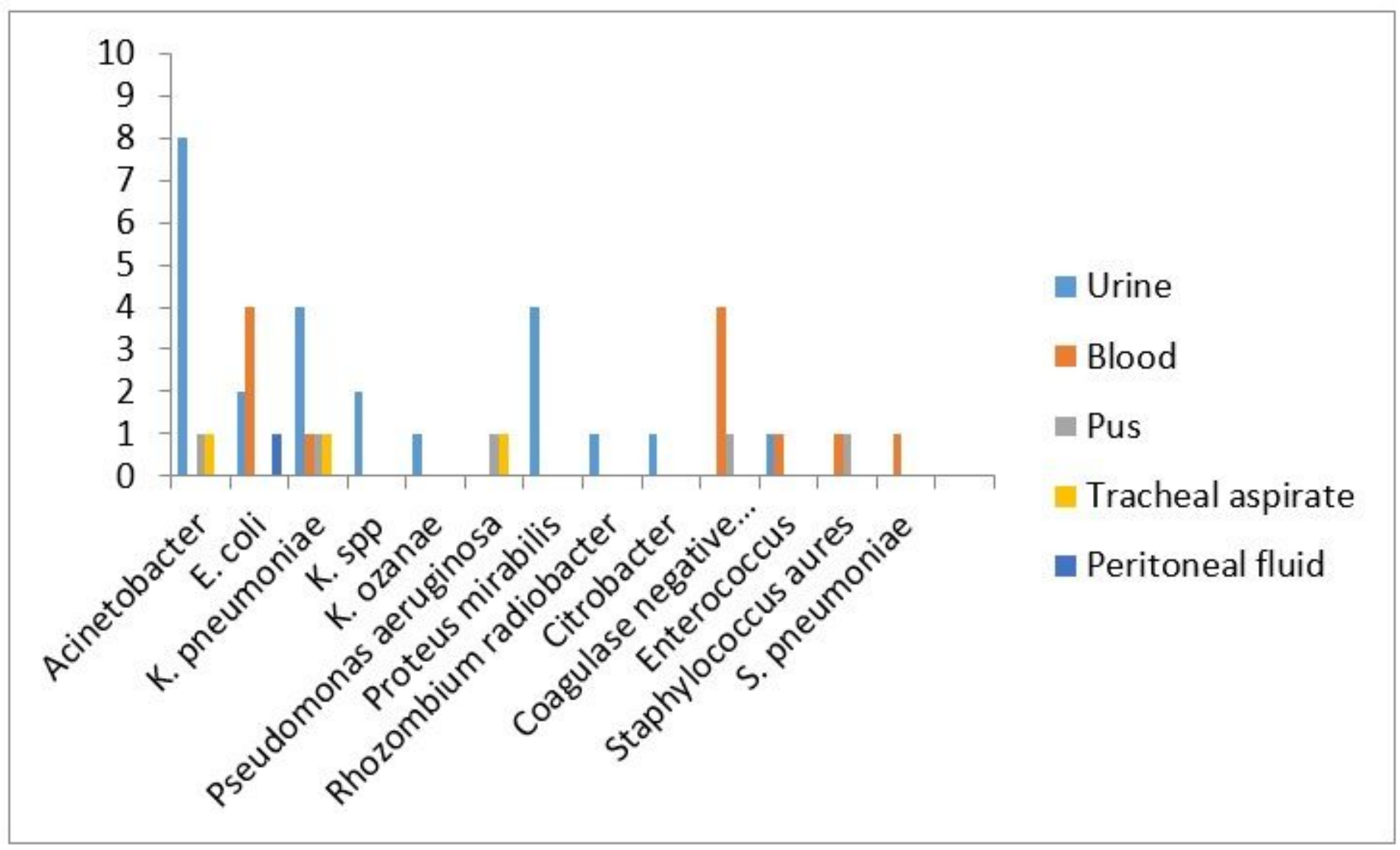


Figure 1

Type of microorganisms grown per type of culture among patients admitted to SPHMMC and AaBET ICUs from January 2019 to December 2019.

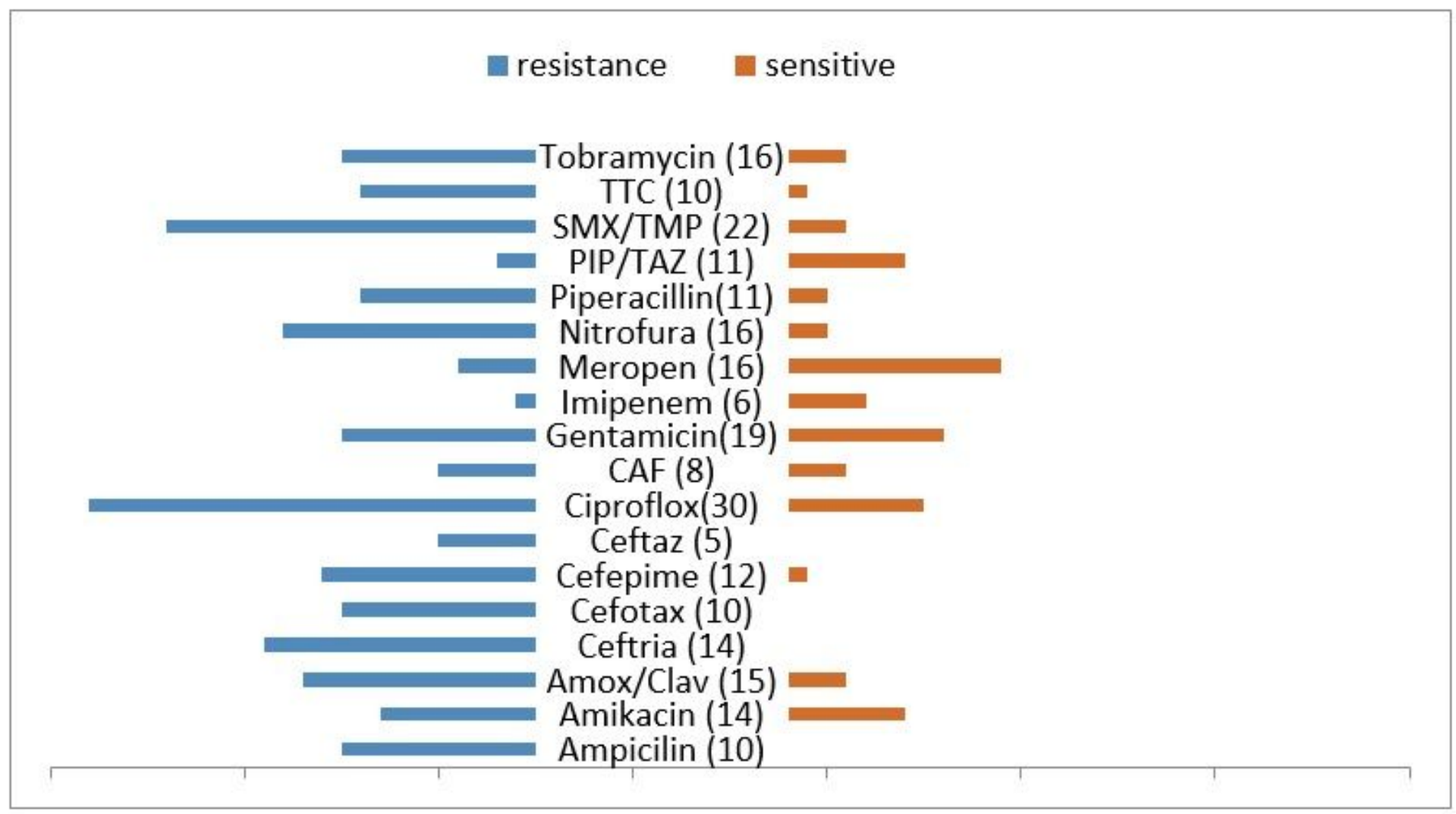

Figure 2

Antimicrobial resistance and sensitivity distribution among patients admitted to SPHMMC and AaBET ICUs from January 2019 to December 2019.

TTC- Tetracycline, SMX/TMP- Sulfmethoxazole/Trimethoprim, PIP/TAZ- Piperacillin/Tazobactam, CAFChloramphenicol.

\section{Supplementary Files}

This is a list of supplementary files associated with this preprint. Click to download.

- Supplementarymaterial.docx 